\title{
"NISSEKI LC Film" Liquid Crystalline Polymer Film for Optical Devices
}

\author{
Takehiro Toyooka and Yoshihiro Kobori \\ Infomaterials \& Devices $R \& D$ Group \\ Central Technical Research Laboratory, Nippon Mitsubishi Oil Corp. \\ 8, Chidori-cho, Naka-ku, Yokohama 231-0815, Japan
}

\begin{abstract}
"NISSEKI LC Film" is a liquid crystalline polymer film, which has unique optical properties as well as excellent reliability and thermal stability. There are two types of film, one has a twisted nematic structure and another has a hybrid nematic structure. They are suitably used as color compensator of STN-LCD and viewing angle compensator of TFT-LCD, respectively.

Keywords: liquid crystal, LCD, twisted nematic, hybrid nematic, compensator
\end{abstract}

\section{Introduction}

Liquid crystalline polymer (LCP) is unique material that has both advantages of liquid crystal and polymer. Nowadays, LCPs are widely used for high strength fibers and heat resistant materials. But in such application, we use a few advantages of LCPs. The combination of optical anisotropy and molecular orientation fixability enables us to make novel optical films. But, to our knowledge, many trials to make optical devices using LCPs are not sufficient, because it is hard to obtain monodomain LCP films. We have challenged producing mono-domain LCP films and have established the technology to produce optical grade mono-domain LCP films. In 1995, we introduced the world's first of the optical grade mono-domain LCP film that is named as "NISSEKI LC Film". In this paper, we report structure and properties of "NISSEKI LC Film", and also report application to the display device of LC Film.

\section{What is "NISSEKI LC Film"?}

\subsection{Structure of "NISSEKI LC Film"}

Fig. 1 shows cross section of "NISSEKI LC film"(LC-film). LC-film is consisting of liquid crystalline polymer layer that is formed on transparent substrate film and covered with protective layer.[1] Total thickness of LC-film that is mostly dependent on the thickness of the substrate film is a little under one hundred micrometer. On the other hand, optically active LCP layer is a few or less microns, so there is the possibility of very thin optical device in LC-film technology.

As shown in Fig. 2, LC-film is classified to 2 types by orientation of LCP molecules in the LCP

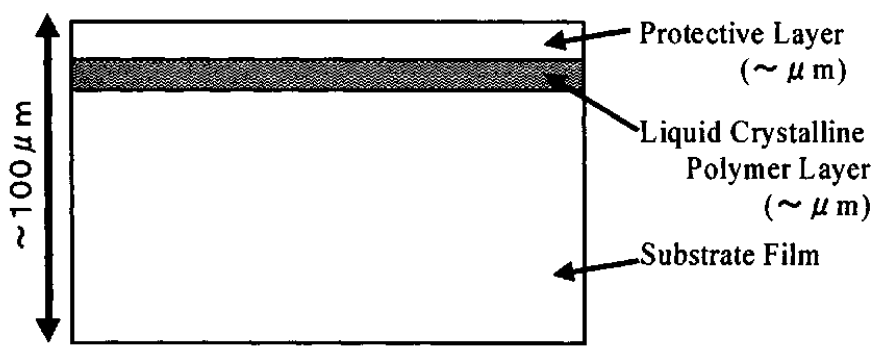

Fig. 1. Structure of "NISSEKI LC Film"

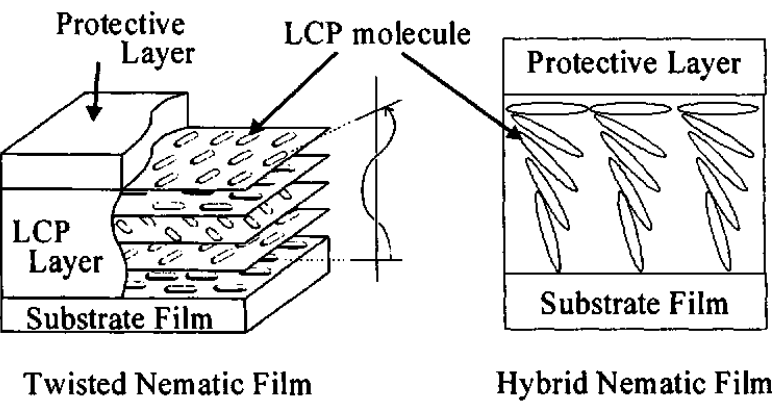

Fig. 2. LCP molecular alignment in "NISSEKI LC Film" 
layer. One is "Twisted Nematic Film" that LCP molecules make helical structure, and another is "Hybrid Nematic Film" that tilt angle of LCP molecule changes in thickness direction of the film. "Twisted Nematic" and "Hybrid Nematic" structure are extremely difficult to produce with stretching technique that is used for the production of ordinary retardation films.

\subsection{Twisted Nematic Film}

Twisted Nematic film has twist angle and retardation as optical parameter. These parameters are controlled freely and independently, and determined by chiral content in the LCP and thickness of the LCP layer. Fig. 3 shows the polarized transmission spectra of the Twisted Nematic Film. The spectra obtained from experiment and calculation show a good agreement. In addition to that, Twisted Nematic Film has high transmission (over 90\%) and low haze (less than $1 \%)$. From these results, we estimate that our film has a uniformly twisted molecular orientation and mono-domain structure.

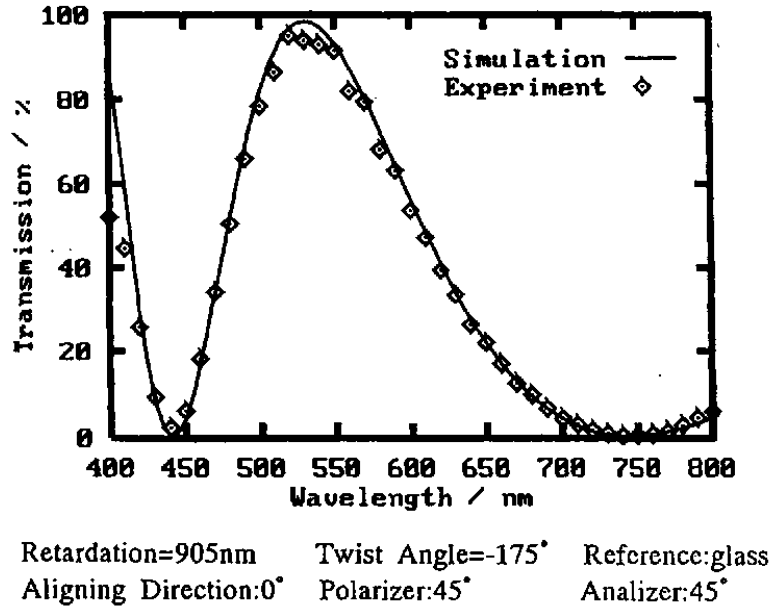

Fig. 3. Polarized transmisson spectra of Twisted Nematic Film.

Not only an optical performance but also stability will become an important factor when we use it for optical devices. Fig. 4 shows the stability of the Twisted Nematic Film under high temperature. The Twisted Nematic Film has high stability, and the optical parameters such as retardation, twist angle and luminous transmittance do not change for over 1000 hours under this condition. Under the condition at high humidity or low temperature, the Twisted Nematic Film is stable. The changes of optical parameters were not seen for over 1000 hours under these conditions.[1]

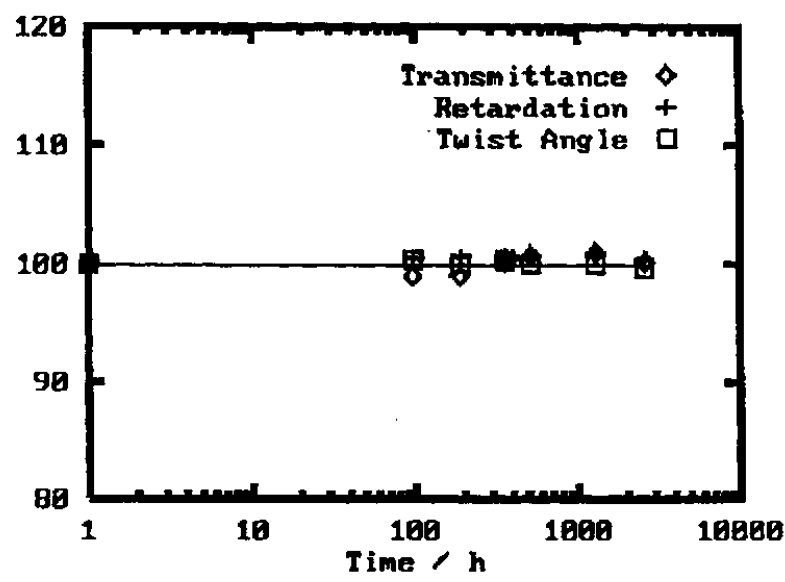

Fig. 4. Thermal stability of optical parameters (transmittance, retardation and twist angle: initial value $=100$ ) in Twisted Nematic Film under the condition of 80 degrees.

\subsection{Hybrid Nematic Film}

In Hybrid Nematic film, LCP molecules are homogeneously aligned at one side of the LCP layer, and those are homeotoropically aligned at the other side. The Hybrid Nematic Film has two optical parameters. One is film thickness, and the other is average tilt angle of LCP molecules. The asymmetric structure of this film is easily estimated by optical measurements. We also confirmed the structure from the angular dependence of retardation of the Hybrid Nematic Film. The optical measurements agreed well with data obtained from calculation based on hybrid

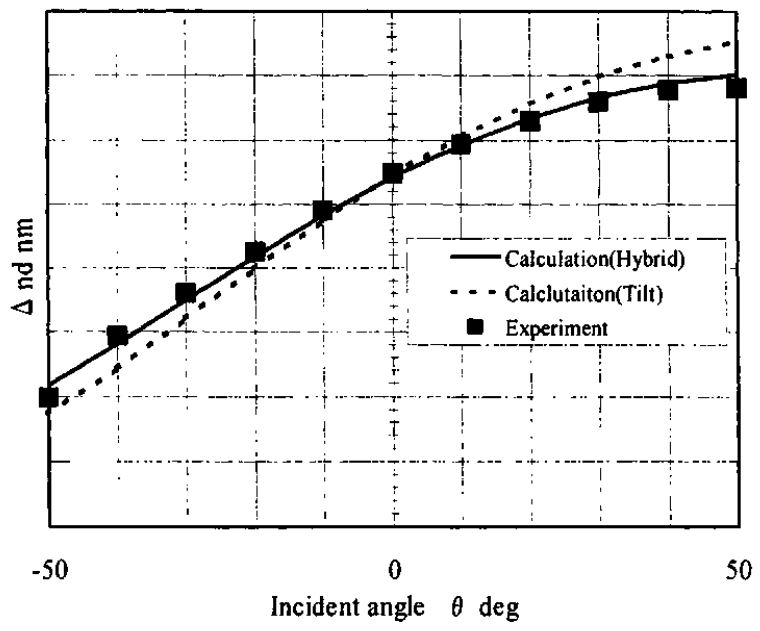

Fig. 5. Dependence of retardation on incident angle. 

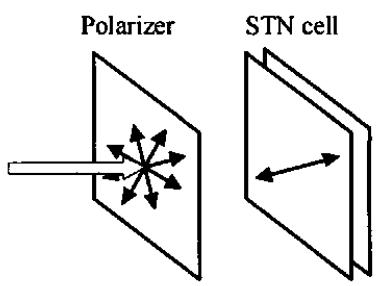

Polarizer
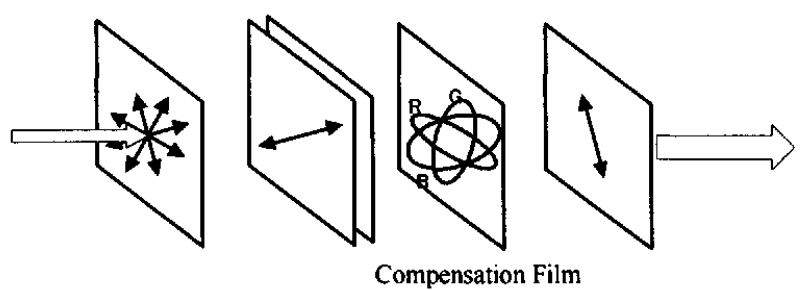

Fig. 6. Principle of color compensation of STNLCD

aligned nematic structure, as shown in Fig. 5. From these observations, we conclude that our film has hybrid aligned nematic structure without twist.[2]

\section{Application to compensator for STN-LCD}

\subsection{Color compensation of STN-LCD}

STN(Super Twisted Nematic)-LCD was developed in the middle of 1980s, and mainly used for mobile applications such as PDA, cellular phone, portable game, etc.[3] As STN-LCD has steeper brightness versus driving voltage characteristic than TN-LCD, STN-LCD enables large capacity display by passive matrix addressing. However, on the principle that the large twist angle and birefringence effect is used, STN-LCD has coloration on displayed images. To overcome this problem, some techniques are proposed.

(1) Adjust the optical parameter of the cell(OMI mode) [4]

(2) Add dye into the liquid crystal material[5]

(3) Optical compensation cell (Double Cell STN)[6]

(4) Optical compensation film[7,8]

In these candidates, optical compensation film is a mainstream now.

The color compensation principle of STN is shown in Fig. 6. The STN cell is located between crossed polarizer. Incident light on STN cell is linearly polarized, and is transformed to the elliptical polarization. The compensation film retransforms this elliptical polarized light to the linearly polarized light. As the major axis and the ellipticity of the polarization differ by wavelength, the Twisted Nematic Film is optimal to complete compensation.

\subsection{Performance of Twisted Nematic Film}

Fig. 7 shows the transmission spectra of STNLCD in black state. In the case that Twisted Nematic Film was used, low and uniform transmission spectrum, in other word "high quality black image " was obtained. However, "perfect black" was not realized, because the wave length dependence of the birefringence of LCP and liquid crystal material used in STN cell are different. As shown in Fig. 8, complete black state is established by matching the wave length dependence of the birefringence of LCP and that of STN cell.[9]

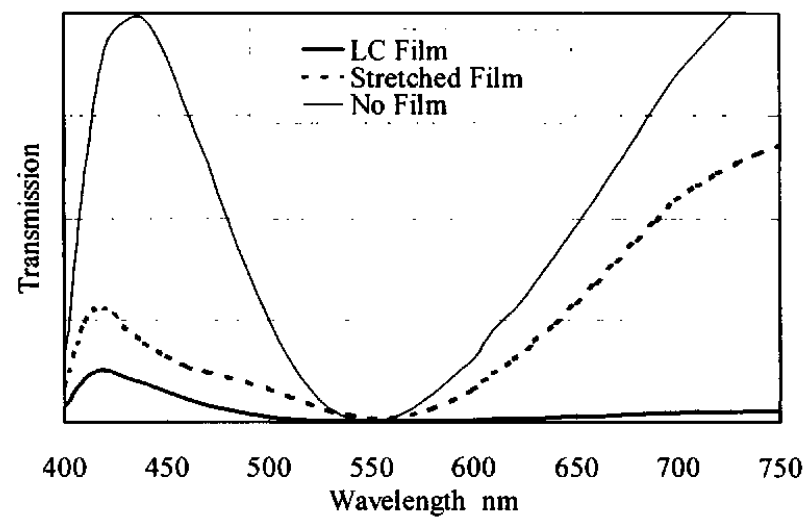

Fig. 7. Transmission spectra of STN-LCD in black state with LC film (heavy line), uniaxially stretched film (dashed line), and without film (solid line).

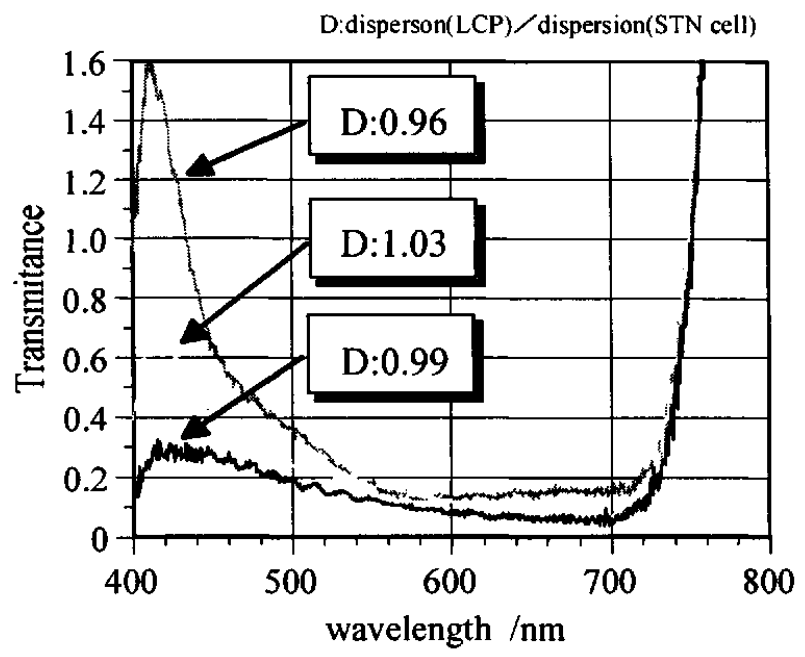

Fig. 8. Black state transmission spectra of compensated STN-LCD by Twisted Nematic Film with various dispersion. 


\section{Application to wide viewing angle TFT-LCD}

\subsection{Viewing angle performance of TFT-LCD}

According to remarkably improvement in performance, TFT-LCD has been widely used for television, car navigation system, video camera, note PC, flat panel monitor for PC and so on. However, viewing angle performance of TFT-LCD is one of the most serious problems to be solved for advanced multimedia application. For the past few years, some new wide viewing angle mode LCDs such as IPS[10], MVA[11] and OCB[12] were developed. These new modes LCDs show good viewing angle performance, but there is a strong demand for improving the viewing angle performance of conventional TN-LCD.[13]

Compensation film technique is very hopeful for improving the viewing angle performance of conventional TN-LCD, and that is very attractive because that needs no changes for conventional LCD manufacturing processes. Nowadays, some kinds of compensation film technique have been proposed. [14,15,16,17]

\subsection{Viewing angle compensation of TN mode}

It is well known that tilted negative birefringence film is adequate for viewing angle compensator of normally-white(NW)-TN-LCD, because that has opposite symmetry to NW-TN cell at black state in which liquid crystal molecule used has positive birefringence, and the directors are tilted from the cell substrate. To obtain the tilted negative birefringence film, some approaches, such as obliquely deposited metal oxides[14], discotic compounds[16] and a stack of two tilted positive layers[17], have been reported. Fig. 9 shows the concept of compensation of NW-TN cell with single layer of tilted positive birefringence film. In the black state of NW-TN cell, the director profile in the interface region near the substrate of the cell is far from homeotropic, whereas that in the middle of the cell is approximately homeotropic but slightly tilted. It is able to produce negative properties by combining the liquid crystal molecules in the interface region of the cell with the tilted molecules of the film. The negative properties thus formed can again compensate the positive properties in the middle of the cell.

\subsection{Performance of Hybrid Nematic Film}

Hybrid Nematic Film improves the viewing angle performance. Fig. 10 compares the measured Iso-CR curve between a compensated TN cell and

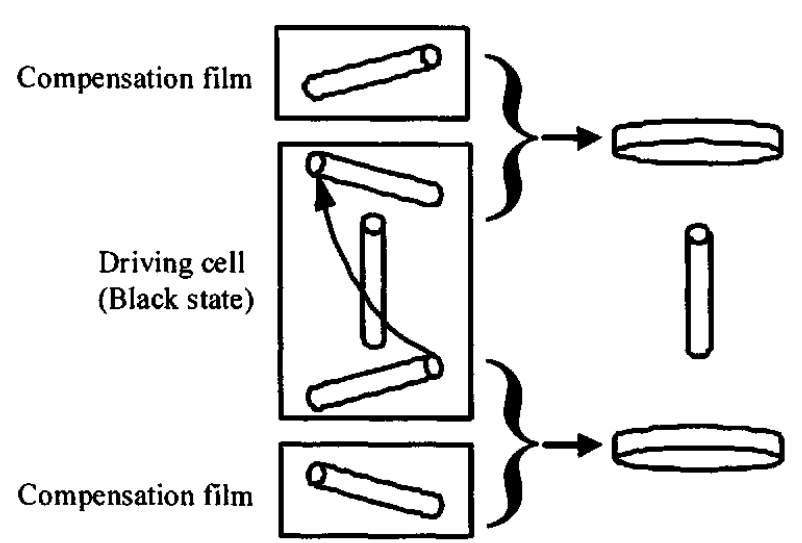

Fig. 9. Concept of compensation for NW-TNLCD with tilted positive films.

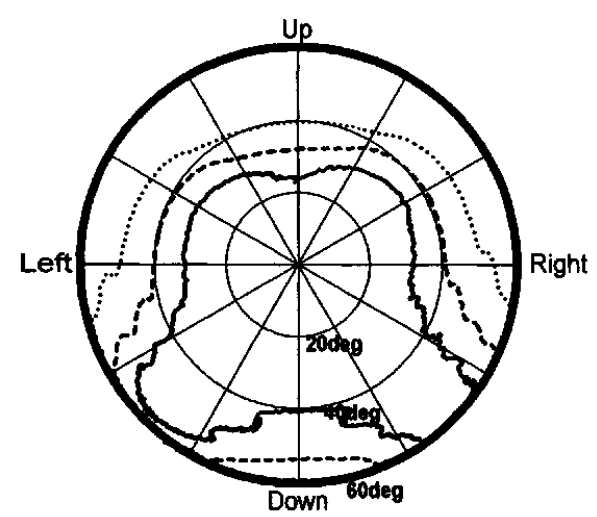

(a) with LC Film

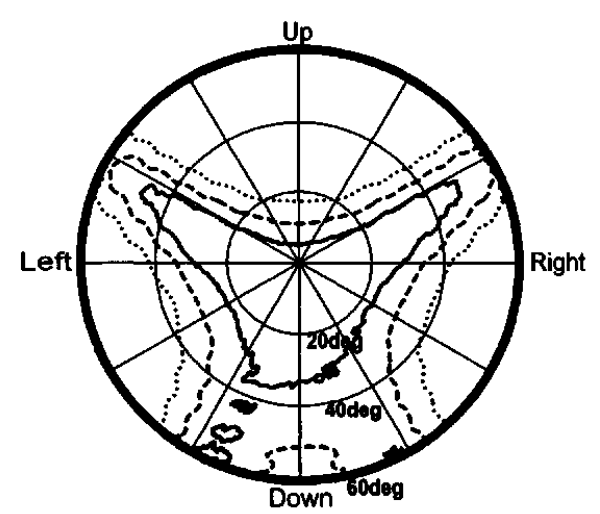

(b) no film(conventional)

Fig. 10. Iso-contrast curves of compensated (a) and conventional (b) TN-LCD. ( $C R=100$ : solid line, $\mathrm{CR}=30$ : dashed line, $\mathrm{CR}=10$ : dotted line)

a conventional one. Contrast 10 ranges of the compensated LCD become over 90 degrees in vertical direction and over 100 degrees in horizontal direction. Moreover, we can realize wider viewing angle performance by optimizing the parameters of the compensation film or/and the driving cell.[18] 


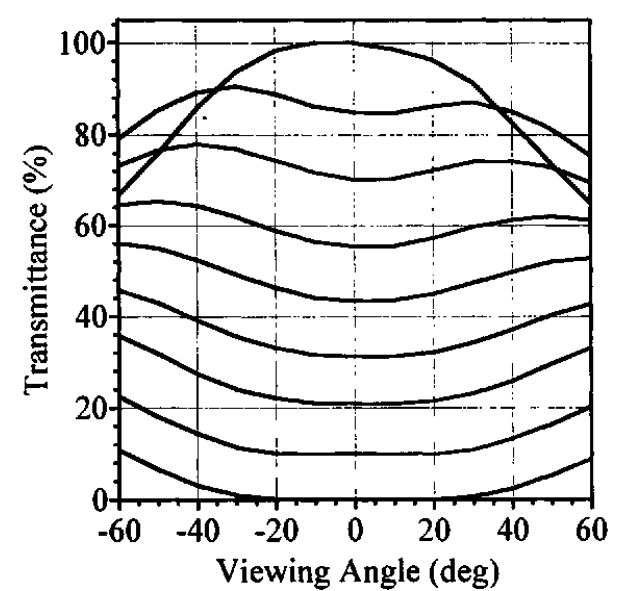

(a) With LC Film (horizontal)

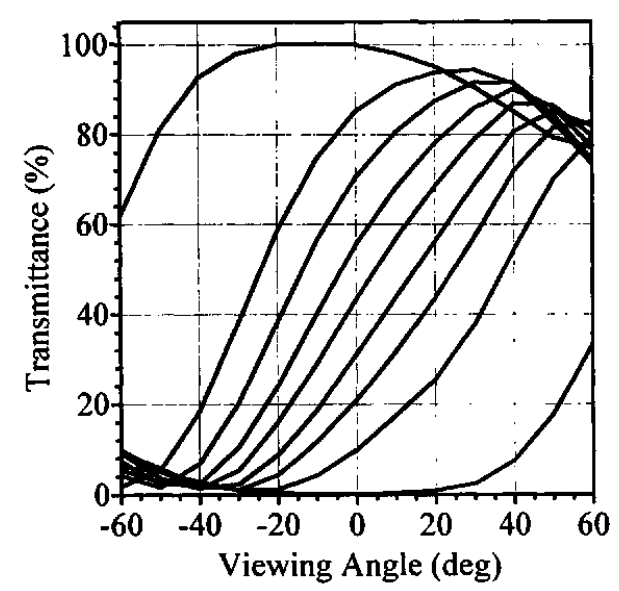

(c) With LC Film (vertical)

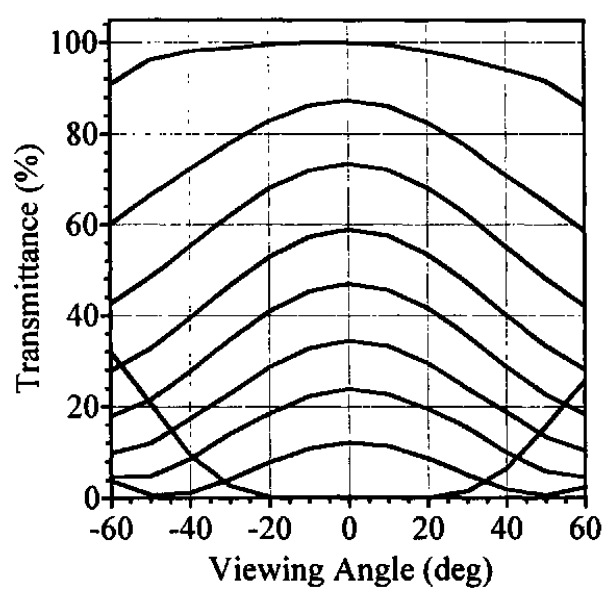

(b) no film(horizontal)

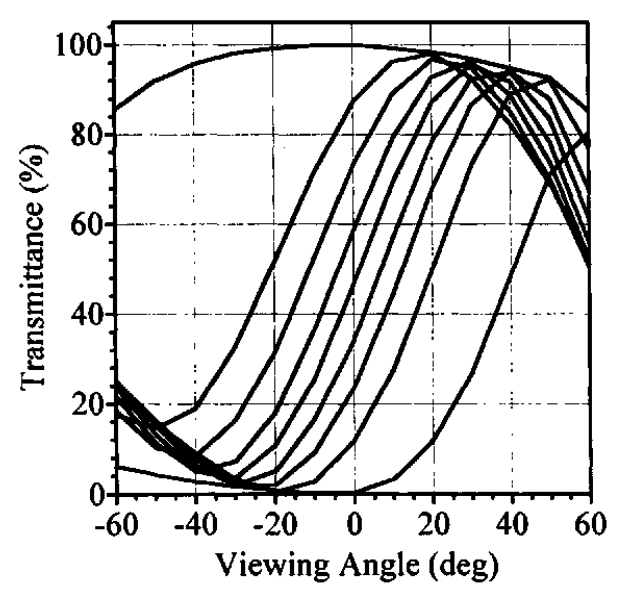

(d) no film(vertical)

Fig. 11. Viewing angle dependence of transmission of compensated (a),(c) and conventional (b),(d) TN-LCD at 8 gray levels in horizontal and vertical direction

Our film enables not only enlargement of high $\mathrm{CR}$ region, but also reduction of inverse region, where black level becomes brighter than gray levels. TN-cell with the compensation film maintains the relationship of each gray level for normal incidence even in large viewing angle. The inversion in white level is improved by controlling the driving voltage on white level.[18] Furthermore, in horizontal direction, the compensated TNcell gives brighter images compared with conventional one, because the transmission shows only a slight dependence with viewing angles, as shown in Fig. 11.[19]

Fig. 12 compares displayed image on both the compensated and the conventional TN-LCD. The TN-LCD that used Hybrid Nematic Film shows a fine viewing angle performance with all directions.

\section{Summary}

We developed a mono-domain, liquid crystalline polymer film named "NISSEKI LC Film". The film has unique optical properties that originate in liquid crystalline molecular orientation. In this paper, we introduced two types of LC Film; one is "Twisted Nematic Film", and another is "Hybrid Nematic Film". The former exhibits excellent performance as an STN-LCD compensator, and the latter enables wide viewing angle TFT-LCD that is suitable for multimedia application.[20] By controlling the various molecule orientation, "NISSEKI LC Film" has the potential to be used in many optoelectronics applications. 


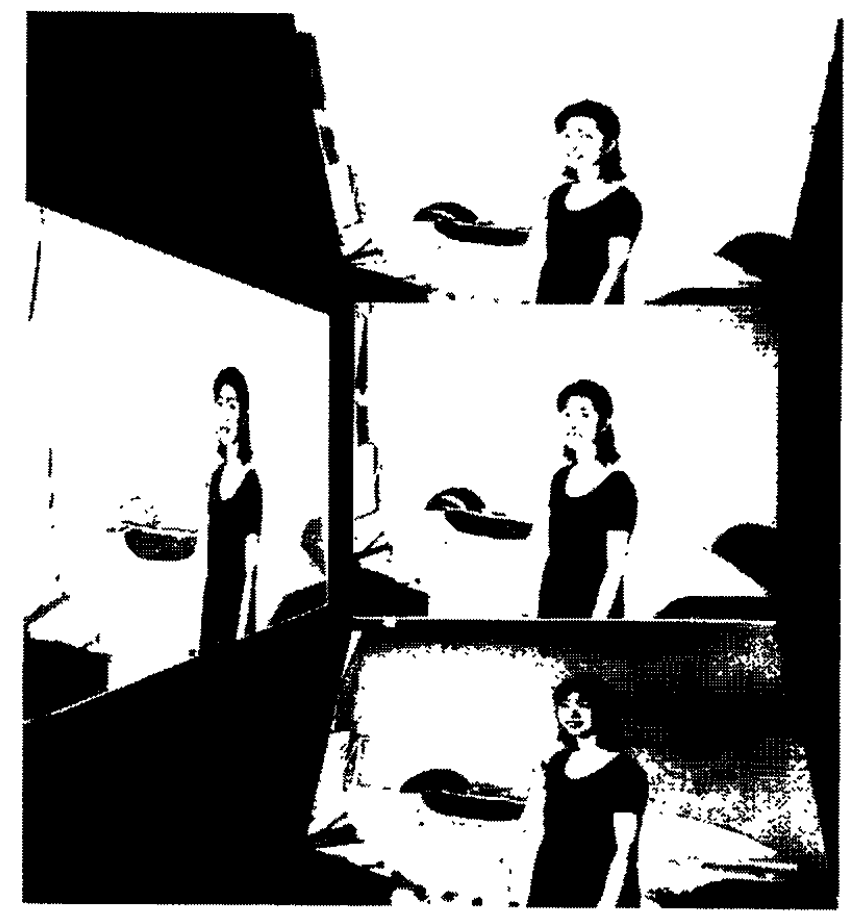

(a) With LC Film

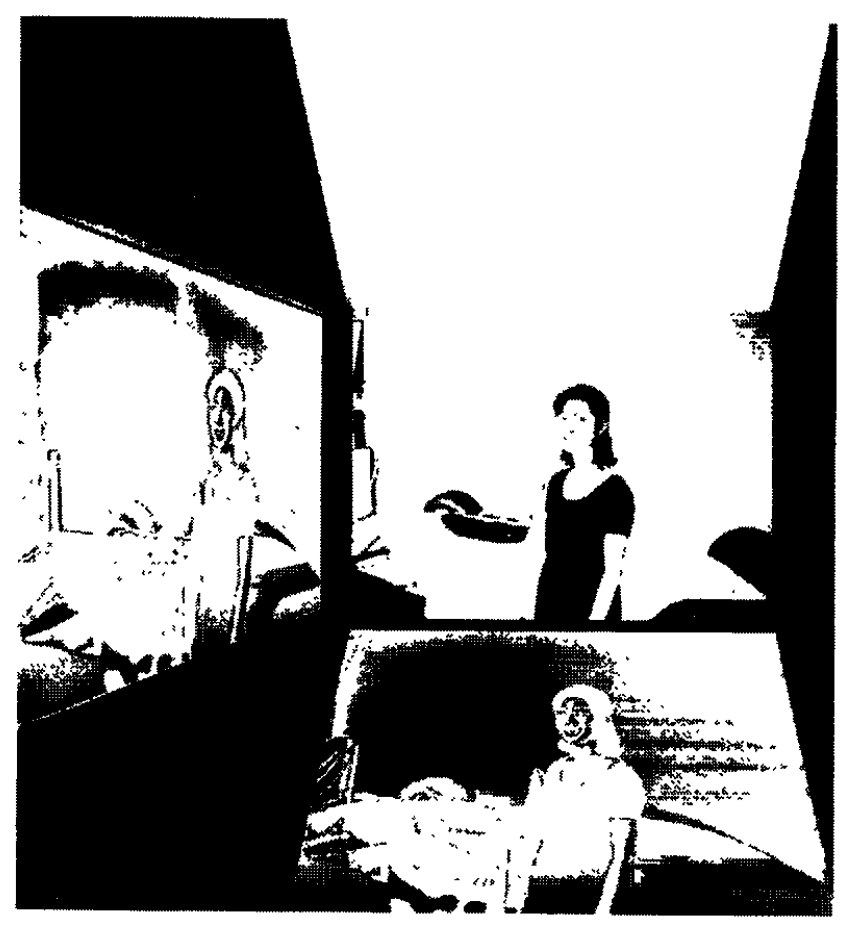

(b) no film

Fig. 12. Viewing angle performance of compensated (a) and conventional (b) TN-LCD.

\section{Reference}

1. J. Mukai, T. Kurita, T. Kaminade, H. Hara, T. Toyooka, H. Itoh, SID '94 Digest(1994) 241.

2. E. Yoda, T. Uesaka, T. Toyooka, Y. Kobori, T. Yamanashi and H. Itoh, Proc. Jpn. Liq. Cryst. Conf. '97 (1997) 128.

3. T. J. Scheffer and J. Nehring, Appl.Phys.Lett. 45(1984) 1021.

4. M. Schadt and F. Leenhouts, Appl. Phys. Lett. 50(5) (1987) 236.

5. K. Kinugawa, Y. Sindo, K. Nagae, Denshi Zairyo 2 (1988) 59.

6. H. Koh, K. Sawada, M. Ohgawara, T. Kuwata, H. Tsubota, M. Akatsuka and K. Matsuhiro, SID '88 Digest (1988) 53.

7. Y. Takiguchi, A. Kanemoto, H. Iimura, T. Enomoto, S. Iida, T. Toyooka, H. Itoh and H. Hara, 10th IDRC

(1990) 96.

8. K. Nagae, J. Hirakata and S. Komura, ITE Technical Report 12(32) (1988) 29.

9. S. Nishimura, T. Matsumoto, T. Toyooka, H. Itoh, T. Satoh, S. Takikawa, SID '95 Digest (1995) 567.
10. M. Ohta, M. Oh-e and K. Kondo, Proc.Asia Display' '95 (1995) 707.

11. K. Ohmuro, S. Kataoka, T. Sasaki and Y. Koike, SID '97 Digest (1997) 845.

12. Y. Yamaguchi, T. Miyashita and T. Uchida, SID '93 Digest (1993) 277.

13. M. Schadt, W. Helfrich, Appl. Phys. Lett., 18 (1971) 127.

14. J. P. Eblen, W. J. Gunning, J. Beedy, D. Taber, L. Hale, P. Yeh, M. Khoshnevisan, SID '94 Digest (1994) 245.

15. J. Mukai, H. Mazaki, Y. Satoh, Y. Kobori, T. Kaminade, T. Toyooka and H. Itoh, Proc. Asia Display '95 (1995) 949.

16. H. Mori, Y. Itoh, Y. Nishiura, T.Nakamura and Y. Shinagawa, Proc. AM-LCD'96/IDW'96 (1996) 189.

17. P. van de Witte, S. Stallinga, J.A.M.M. van Haaren, SID'97 Digest (1997) 687.

18. T. Toyooka, E. Yoda, Y. Kobori, T. Yamanashi, H. Itoh, SID '98 Digest (1998) 698.

19. T. Kaminade, E. Yoda, K. Suzuki, T. Toyooka, Y. Kobori, K. Inoue, T. Kurita, Proc. IDW'99 (1999)

20. K. Inoue, T. Kurita, E. Yoda, T. Kaminade, T. Toyooka, Y. Kobori, Proc. IDW'98 (1998) 225. 